\title{
Point-Line Characterizations of Buildings
}

\author{
Arjeh M. Cohen \\ $C W I$, \\ Kruislaan 413, \\ $1098 \mathrm{SJ}$ Amsterdam, \\ The Netherlands.
}

\section{Introduction.}

A space is a pair $(P, L)$ consisting of a nonempty set $P$, whose members are called points, and a collection $L$ of subsets of $P$ of size at least two, whose members are called lines. A subspace $X$ of a space $(P, L)$ is a subset $X$ of $P$ with the property that each line (in $L$ ) having at least two points of $X$ is completely contained in $X$. The kind of spaces that we are interested in are obtained from a building (cf. Tits [39]) $B$ (say) of type $M$ (say) over an index set $I$ (say) in the following way: Fix a type $i$ (i.e., an element of $I$ ). Let $P$ be the set of objects in the building of type $i$, and let $L$ consist of all subsets of $P$ of the form $P \cap \operatorname{Res}(F)$ (the shadow of $F$ on $P$ ) for some flag $F$ of $B$ of cotype $i$. Such a space will be called the shadow space of $B$ over $i$. Other names for shadow spaces appearing in the literature are Lie incidence system (cf. Shult [36]) and Lie incidence structure (cf. Cooperstein [26]). In these notes, we shall often write $M_{n}$ for $M$ in order to indicate that we have taken $I=\{1,2, \ldots, n\}$. Instead of 'shadow space of a building of type $M_{n}$ over $i$ ' we shall often write shadow space of type $M_{n, i}$. The idea of a shadow space can be found in Tits [39]. His notion of shadow space differs from ours in that it keeps track of other shadows as well.

In the spherical Coxeter diagrams that we shall need, the labelling of the nodes will be as indicated in Figure 1. If, for example, $(P, L)$ is the shadow space of type $A_{n, 1}$, there is a building of type $A_{n}$ associated with a skew field $K$ whose shadow space over 1 coincides with $(P, L)$, so that the latter is nothing but (the set of points and the set of lines of) the projective space of rank (i.e., projective dimension) $n$ over $K$ (cf. Tits [40]). Conversely, if $(P, L)$ is a projective space of rank $n$, the obvious geometry related to $(P, L)$ is the one in which for each $i(1 \leqslant i \leqslant n)$ the objects of type $i$ are the subspaces of rank $i-1$ (incidence being symmetrized containment). It is a building of type $A_{n}$ and $(P, L)$ is (isomorphic to) the shadow space of this building over 1 , whence of type $A_{n, 1}$.

Our aim stems from classical synthetic geometry: to axiomatically describe these spaces in such a way that, on the basis of simple properties (axioms), the geometry can be fully analyzed and recognized. The first result of this kind is:

\subsection{Theorem(Veblen \& Young).}

Let $(P, L)$ be a space in which every two (distinct) points are on a unique line. Suppose that, for each foursome of lines, if (at least) five out of the six pairs of lines intersect in distinct points, all pairs meet nontrivially. Then $(P, L)$ is a (generalized) projective space. 


$$
\left.A_{n}:=12 \geqslant 1\right)
$$

Figure 1.

In Shult [36], an excellent description of the goals of synthetic Lie geometry and a survey of recent progress in the field can be found. The reader will notice some overlap between Shult's survey and the present notes.

Let $(P, L)$ be a shadow space of $B$ over $i$. The associated full shadow space is the space endowed with, for each $j \in I$, the collection of subsets $P \cap \operatorname{Res}(F)$ (the shadow of $F$ on $P$ ) where $F$ ranges over all flags of type $j$. Thus, for type $A_{n, 1}$, the full shadow space is the projective space together with, for each $i(1 \leqslant i \leqslant n)$, the collection of subspaces of rank $i-1$. The natural question as to whether the shadow space does determine the building will be answered by use of these full spaces as a link between shadow spaces and buildings. A similar pattern can be found in the proof of the characterization theorems we shall describe: starting from a space, subspaces of various kinds mimicking the shadows of the full shadow space are collected in order to construct a geometry with diagram $M$. Then by the basic Proposition 9 in Tits [40] (see also Brouwer-Cohen [5]), the geometry in question can be identified as a building. As a matter of fact, sometimes information weaker than the full shadow space is available. In such cases, due to a slight generalization of Tits' result on truncated geometries (cf. Ronan [32] and Brouwer-Cohen 
[6]), it is still possible to identify the space with a quotient of a shadow space by a group of automorphisms (with certain well-described properties).

Before ending the introduction, I would like to emphasize that the analogue of the coordinatization theorem of projective spaces for (other) buildings, established by Tits and Veldkamp (cf. Tits [39]), and the subsequent classification of geometries with spherical Coxeter diagram satisfying the 'intersection property' (cf. Tits [40]), are basic prerequisites to the theorems of the sequel: without them the results presented here would have little meaning.

\section{Some properties of shadow spaces.}

In order to be able to choose natural axioms for shadow spaces we need a list of at least some of their characteristic properties.

First, some generalities on spaces. A subset $X$ of $P$ is called complete or singular if each pair of points from $X$ is collinear. The singular rank of a subspace $X$ of $(P, L)$ is the maximal length of a chain of nonempty complete subspaces contained in $X$, where the length of a chain is one less than its cardinality. Thus, the singular rank of the empty space, a point, a line is $-1,0,1$, respectively. Also, the singular rank of a projective space is just its rank. (All subspaces are singular!) A subset of $P$ is called degenerate if it contains a point collinear with all of its points. The space $(P, L)$ itself is called degenerate if $P$ is degenerate. The collinearity graph of a space $(P, L)$ is the graph $(P, \perp)$ with vertex set $P$ in which $x \perp y$ holds if and only if $x$ and $y$ are collinear points of $P$. Notions such as connectedness, adjacency, diameter, distance, path, when applied to $(P, L)$, are meant to refer to $(P, \perp)$. The distance is denoted by $d$. A subset $X$ of $P$ is called geodesically closed if, for all $x, y \in X$, each point on a path of minimal length from $x$ to $y$ is also contained in $X$. If $X$ is a subset of $P$, we denote by $X^{\perp}$ the set of all points in $P$ collinear with every member of $X$. Often, $\{x\}^{\perp}$ is replaced by $x^{\perp}$.

\subsection{Theorem(Brouwer-Cohen).}

Suppose $B$ is a building of type $M=\left(m_{j, k}\right)_{j, k \in I}$ over $I$. Let $i \in I$, and let $(P, L)$ be the shadow space of $B$ over $i$. The following statements hold for $(P, L)$ :

i) Each pair of distinct points is on at most one line. Either there is a unique $j \in I$ with $m_{i, j}=3$ and there is a connected component of the restriction of $M$ to $\left\{k \in I \mid m_{i, k}=2\right.$ or 3$\}$ isomorphic to $A_{m}$ for some $m$ containing $j$ as an end node, or $\{x, y\}^{\perp \perp}$ is the unique line containing $x, y$ for every pair $x, y$ of collinear points.

ii) Every shadow $X$ is a geodesically closed subspace. As a space, $X$ is the shadow space over $i$ of a building isomorphic to $\operatorname{Res}(F)$ where $F$ is a flag of $B$ which is maximal with respect to the property that its shadow on $P$ coincides with $X$.

iii) For each shadow $X$ and point $x$ the subset

$$
\pi_{x}(X):=\left\{y \in X \mid d(x, y)=\min _{z \in X} d(x, z)\right\}
$$

of $P$ is a subspace. Hence, $x^{\perp}$ and $X^{\perp}$ are subspaces.

iv) If $x, y \in P$ satisfy $d(x, y)=2$, then either $\{x, y\}^{\perp}$ is a subspace isomorphic to a projective space (if $M$ is spherical, necessarily of rank 0 ) or $\{x, y\}^{\perp} \cup\{x, y\}$ is contained in a shadow subspace of type $B_{m, 1}$ (or $D_{m, 1}$ ) for some $m>1$,

$v$ ) The shadow of an apartment of $B$ (i.e., the set of points belonging to an apartment) induces $a$ subgraph of $(P, \perp)$ which is isomorphic to the shadow space of the thin building of type $M$ over $i$. 
About the proof. The proof is based on the following lemma about Coxeter groups. The reader is referred to Bourbaki [4] for the definitions of Coxeter system (p.11) and $(J, J)$-reduced element (p.37), where $J \subset R$. If $w \in W$, we shall denote by $R_{w}$ the set of all elements in $R$ occurring in a shortest expression of $w$ as a product of elements from $R$ (cf. p.19 in [4]).

\subsection{Lemma.}

Let $(W, R)$ be a Coxeter system of type $M$. Fix $r \in R$. Set $J=R-\{r\}$ and $J_{k}=\left\{s \in J \mid m_{r, s}=k\right\}$, for $k \in \mathbb{N}$. Then the following hold.

i) If $W=\langle J\rangle r\langle J\rangle$, then $M=A_{n}$ for some $n$, and $r$ is an end node of $M$.

ii) $r<J>r \cap<J>=\left\langle J_{2}>\right.$ and $\left.r<J>r<J>\cap<J>r<J>=<J_{2}>J_{3} r<J\right\rangle$.

iii) Let $K$ be a subset of $J$. If $w_{0}, w_{1}, \ldots, w_{k} \in<J>$ satisfy $w=w_{0} r w_{1} r \cdots r w_{k} \in<K \cup\{r\}>$ and $d(\langle J\rangle, w<J>)=k$ (in the shadow space of the thin building over $r$ ), then $w_{0} r w_{1} r \cdots r w_{j} \in<K \cup\{r\}><J>$ for each $j(0 \leqslant j \leqslant k)$.

iv) Let $w \in W$ be $(J, J)$-reduced and $d(w<J>,<J>)=2$. Set $T=R_{r w r} \cap w R_{r w r} w^{-1}$. Then $\langle J>r<J>\cap w<J>r<J\rangle=\left\langle T>r<J>\right.$. Moreover, either $T \subseteq J_{2}$ or $r w r \in J_{4} \cup J_{3}<J_{2}>J_{3}$.

The proof of the lemma will be published elsewhere.

As for the theorem, the following verification of the first statement may illustrate the kind of arguments involved in the proof. We shall identify $R, r$ (of the lemma) with $I, i$.

Let $x, y \in P$ be distinct. We adopt the chamber system point of view: $x, y$ are distinct $J$-cells and there are chambers $a \in x, b \in y$ contained in the same $r$-cell $F$, say. Then $P \cap \operatorname{Res}(F)$ is a line on $x$ and $y$. Now, let $F^{\prime}$ be another $r$-cell meeting $x$ and $y$ in chambers $a^{\prime}, b^{\prime}$, say, respectively. For any two chambers $c, c^{\prime}$, denote by $\operatorname{typ}\left(c, c^{\prime}\right)$ the element of the Coxeter group $W=W(M)$, corresponding to the type of a minimal gallery joining $c$ to $c^{\prime}$, cf. Tits [40]. Since appending $b^{\prime}$ to a minimal gallery from $a$ to $a^{\prime}$ yields a minimal gallery from $a$ to $b^{\prime}$, we have $\operatorname{typ}\left(a, b^{\prime}\right)=\operatorname{typ}\left(a, a^{\prime}\right) r$. Similarly, using a gallery passing through $b$, we obtain typ $\left(a, b^{\prime}\right)=r t y p\left(b, b^{\prime}\right)$. As typ $\left(a, a^{\prime}\right), \operatorname{typ}\left(b, b^{\prime}\right) \in\langle J\rangle$, it follows from Part ii) of the lemma that $\operatorname{typ}\left(a, a^{\prime}\right)=\operatorname{typ}\left(b, b^{\prime}\right) \in\left\langle J_{2}\right\rangle$. Now suppose that $z$ belongs to $P \cap \operatorname{Res}(F)$. Then there is a chamber $c \in z \cap F$, so $\operatorname{typ}\left(c, b^{\prime}\right)=r \operatorname{typ}\left(b, b^{\prime}\right)=\operatorname{typ}\left(b, b^{\prime}\right) r$. By Tits [40], there must be a minimal gallery from $c$ to $b^{\prime}$ whose one but last chamber, $c^{\prime}$, say, satisfies typ $\left(c, c^{\prime}\right)=\operatorname{typ}\left(b, b^{\prime}\right) \in\langle J\rangle$ and $\operatorname{typ}\left(c^{\prime}, b^{\prime}\right)=r$. Hence, $c^{\prime} \in z \cap F^{\prime}$, so that $z \in P \cap \operatorname{Res}\left(F^{\prime}\right)$. Since the roles of $F$ and $F^{\prime}$ can be interchanged in the previous argument, $P \cap \operatorname{Res}(F)$ and $P \cap \operatorname{Res}\left(F^{\prime}\right)$ coincide, proving that there is a unique line on $x$ and $y$.

Let us briefly discuss the proof of the remaining parts of the theorem. The second half of Part $i$ ) is a consequence of Part $i i$ ) of the lemma. ( Observe that, in the second equality of $i i$ ), the left hand side is the union of all cosets pertaining to $l^{\perp \perp}-l$, where $\left.l=\{\langle J\rangle, r<J\rangle\right\}$, while the right hand side provides an explicit description of this set.) The spherical case is due to Cooperstein [25]. The first statement in ii) follows from Part iii) of the lemma, where the geodesical closure of the shadow of a flag of type $R-(K \cup\{r\})$ on $P=W /\langle J\rangle$ is formulated. The second statement in $i i$ ), as well as Parts $i i i)$ and $v$ ), are well-known properties of buildings and easily derived by use of the chamber system point of view. Statement $i v$ ) depends on Part $i v$ ) of the lemma, where, for the points $W_{J}, w W_{J}$ at mutual distance 2 in the shadow space of the thin building, a description of the points collinear with both $\langle J\rangle$ and $w\langle J\rangle$ in $P$ is given. As a matter of fact, by use of induction on the length of $w$ (with respect to $R$ ), the following more precise information on $\langle T\rangle r\langle J\rangle$ can be obtained:

Set $s_{0}=r$. Either $T=\varnothing$ or there are $s_{1}, \cdots, s_{q}, s, t \in J, u \in<R_{w}>$, and $x \in \mathbb{N}, x \geqslant 2$, such that $m_{a, b}=2$ for all $a \in T_{0}:=T \cap R_{u}$ and $b \in\left\{s_{0}, \cdots, s_{q}, s\right\}$, and such that $w,\left.M\right|_{R_{u}}-R_{u}$, and 
$\langle T>r\langle J\rangle$ are as given in one of the rows of the following table. Moreover, $u$ is $\left(R_{w}-R_{u}, R_{w}-R_{u}\right)$-reduced, and if $u=1$, then $x>2$.

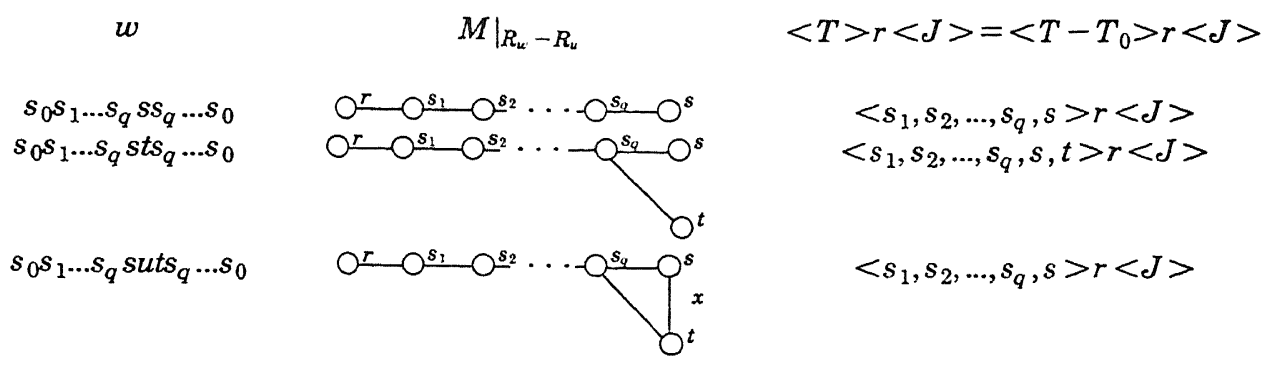

The first two lines represent the cases where $\{x, y\}^{\perp \perp}$ is a polar space (possibly of rank 0 ), in the last case the subset is a complete subspace.

\section{Reconstruction of the building from a space.}

Due to Property $(O)$ in Tits [40], the full shadow space uniquely determines the set of objects of the building. Thus, in order to reconstruct the building from the shadow space, it suffices to reconstruct the full shadow space and incidence between shadow subspaces.

\subsection{Theorem.}

Let $B$ be a building of spherical type $M$ over $I$, and let $i \in I$. Suppose $(P, L)$ is the shadow space of $B$ over $i$. Then, up to not necessarily type-preserving automorphisms of $B$ preserving $i$, the building $B$ of type $M$ can be uniquely reconstructed from $(P, L)$. In other words, if $B^{\prime}$ is another building of type $M$ whose shadow space over $i$ coincides with $(P, L)$, then there is an automorphism $\alpha$ from $B$ to $B^{\prime}$ with $\alpha\left(t y p^{-1}(i)\right)=t_{y p^{\prime-1}}(i)$, where typ and typ' denote the type maps of the respective buildings (cf. Tits [40]).

\subsection{Remarks.}

i) A space can be the shadow space of two buildings of different type. Classical examples are spaces of type $D_{n, 1}, B_{4,2}, D_{4,2}$, which are also shadow spaces of type $B_{n, 1}, F_{4,1}$, and $F_{4,1}$, respectively (cf. Tits [39], Sections 7.12 and 10.14).

ii) The theorem is probably true for many buildings of nonspherical type as well.

Sketch of proof. The problem is to recover the full shadow space from $(P, L)$. Incidence between shadow subspaces is then a matter of having the right intersection.

If the rank of the building is at most two, there is nothing to prove. Subspaces isomorphic to shadow spaces of type $A_{m, 1}$ for some $m$, can be obtained as complete subspaces (and vice versa). Shadow subspaces of type $B_{m, 1}$ or $D_{n, 1}$ are maximal nondegenerate geodesically closed subspaces of diameter 2 (and vice versa). These two observations lead to sufficiently subspaces if the type in question is $A_{n, 1} \quad(n \geqslant 1), B_{n, 1} \quad(n \geqslant 2), D_{n, 1} \quad(n \geqslant 4), F_{4,1}$, or $E_{n, 1} \quad(n=6,7,8)$. If the type is $A_{n, j}$ $(2 j+1 \neq n \geqslant 3), B_{n, n}(n \geqslant 3)$, or $D_{n, n}(n \geqslant 5)$, for each type, the shadows are the geodesical closures of two points at given distance (the type of the shadow only depending on the distance). If the type is $B_{n, n-1}$, one can recover the collinearity graph of $B_{n, 1}$, by letting the point set consist of all maximal complete subspaces of rank $n-1$ and letting two such spaces be collinear whenever 
they have a point in common. By what we have seen before, there is a unique building of type $B_{n}$ (up to isomorphism) associated with this space. This must be the unique building associated with $(P, L)$ as well. Similar reduction arguments apply to many other cases. We shall treat the hardest case, which occurs when the type is $F_{4,2}$. Let $(P, L)$ be a shadow space of this type. Consider the following relations on $P$, where $x, y \in P$ :

a) $d(x, y)=2$ and $\{x, y\}^{\perp}$ has size at least two.

b) $d(x, y)=3$ and every line on $y$ contains a point $z$ such that $x, z$ are in relation a).

Denote by \# the union of $\perp$ and these two relations, and, given two points $x, y \in P$ in relation a) or b), denote by $p(x, y)$ the set of all points in relation \# to both $x$ and $y$. Then $p(x, y)$ is a subspace. Let $P_{1}$ be the collection of all subspaces $p(x, y)$ obtained in this way, and define $\perp_{1}$ on $P_{1}$ by $p \perp_{1} q$ for $p, q \in P_{1}$ if and only if $p \cap q$ contains a point of $P$. Then $\left(P_{1}, \perp_{1}\right)$ is the collinearity graph of the shadow space of a uniquely determined building $B$ (say) of type $F_{4}$ over 1 , and $(P, L)$ can be regarded as the set of lines and the set of pencils (a pencil being the collection of all lines contained in a given complete subspace of rank 2 and containing a given point of that subspace), so $(P, L)$ must be the shadow space of $B$ over 2 (up to isomorphism).

We shall finish by discussing one remaining difficulty: the case where isomorphic shadow subspaces of distinct types occur. By way of example, let us consider type $E_{6,4}$. (The types $A_{2 m+1, m}$ $(m \geqslant 1)$ and $D_{n, n-2}(n \geqslant 4)$ can be dealt with by similar observations.) The two kinds of maximal complete subspaces of rank 4 (corresponding to types 2 and 5, respectively) can be distinguished as the parts of the bipartite graph whose vertex set consists of all complete subspaces and in which two vertices are adjacent whenever their intersection has rank 2 . It will be transparent how the non-type-preserving automorphisms may arise: they interchange the types of the two parts.

\section{Polar spaces.}

Let $(P, L)$ be a space. If $(P, L)$ is a shadow space, a special instance of Theorem 2.1 reads

If $x \in P$ and $l \in L$ then $x^{\perp} \cap l=\varnothing$, a singleton, or $l$.

If we demand that, for each $x$ and $l$, this set is nonempty, we obtain the already classical Buekenhout-Shult Axiom:

If $x \in P$ and $l \in L$ then $x^{\perp} \cap l=$ a singleton or $l$.

A space in which this axiom holds is called a polar space. The rank of a polar space $(P, L)$ is by definition $(1+$ the singular rank of $P$ ).

4.1. Theorem(Buekenhout-Shult).

A nondegenerate polar space of finite rank $n$ is a shadow space of type $B_{n, 1}$.

For a proof, see Buekenhout-Shult [15], where the requirement that lines have length at least three may be dropped.

The Buekenhout-Shult axiom forces the diameter of the space in question to be at most two provided its rank is at least two. (A polar space of rank one has no lines!) In view of a recent result of Johnson \& Shult [30], however, the axiom can be weakened in such a way that no restriction on the diameter is apparent: 
4.2. Theorem(Johnson-Shult).

Let $(P, L)$ be a connected space such that, for each $x \in P$, the set $x^{\perp}$ is a subspace which is a polar space of finite rank at least 3 . Then $(P, L)$ is a polar space of finite rank.

Here, we encounter local recognizability. Suppose $(P, L)$ is a space with the property that $x^{\perp}$ is a subspace whose isomorphism type $T$ (say) does not depend on $x \in P$. Then, $(P, L)$ is called locally recognizable if it is the unique space (up to isomorphism) with the property that $x^{\perp}$ belongs to $T$ for all of its points $x$. Putting the above two theorems together, we see that shadow spaces of thick buildings of type $B_{n}$ or $D_{n}(n \geqslant 3)$ over 1 have this property. Clearly, generalized quadrangles, generalized hexagons and so forth are not locally recognizable.

\section{Parapolar spaces.}

Using polar spaces as building blocks, Cooperstein [26] devised an axiom enabling him to recover all geodesically closed shadow subspaces which are polar spaces. Starting point is a particular instance of Theorem 2.1. A space $(P, L)$ is called a parapolar $k$-space if it satisfies the following three axioms.

i) For each $l \in L$, the (collinearity) graph on $l^{\perp}$ is not complete.

ii) For each $x \in P$, the subset $x^{\perp}$ is a subspace of $P$.

iii) If $x, y \in P$ with $d(x, y)=2$, then $\{x, y\}^{\perp}$ is either a singleton or a polar space of rank $k-1 \geqslant 2$.

A space is called a parapolar space if it satisfies the first two axioms and the third without the requirement that the rank be $k-1$. (It is still required that the rank be at least two.) This definition differs from the one given in Cohen-Cooperstein [24] in that the assumptions on line length and connectedness are dropped. Other variations of parapolar spaces can be found in Buekenhout [9] and Hanssens [29] under the name 'polarized space'.

All shadow spaces of 'spherical' type $\neq A_{n, 1}, B_{n, n-1}, F_{4,2}$ are parapolar spaces. (This is a consequence of Theorem 2.1.)

\subsection{Theorem(Cooperstein).}

Let $(P, L)$ be a parapolar space. Then, every quadrangle (i.e., four points of $P$ on which the induced collinearity graph is a quadrangle) is contained in a unique geodesically closed subspace $S$ isomorphic to a nondegenerate polar space. Moreover, complete subspaces of $(P, L)$ are projective spaces. If $(P, L)$ is a parapolar $k$-space, the rank of $S$ (as a polar space) is $k$.

For a proof, see Buekenhout [9] or Cohen [20], where the subspaces $S$ of the theorem are called hyperlines and symplecta, respectively. Here, we shall adhere to the latter name.

In order to recognize shadow spaces, we need to fix one more parameter. For instance:

$(F)_{J}$ For each symplecton $S$ and each point $x \in P-S$, the rank of $x^{\perp} \cap S$ is a member of $J$.

Observe that, due to the geodesical closure of $S$ and Theorem 2.1, the set $x^{\perp} \cap S$ is a complete and hence projective space so that its singular rank coincides with its (projective) rank. Though Axiom $(F)_{J}$ can be rephrased in terms of points and lines, we have chosen the present formulation in order to emphasize its uniform nature. 
5.2. Theorem(Cohen-Cooperstein, Brouwer-Cohen).

Let $(P, L)$ be a connected parapolar $k$-space $(k \geqslant 3)$ of finite singular rank all of whose lines have at least three points and in which $\left(F_{\{-1,0, k-1\}}\right)$ holds. If the size of $\{x, y\}^{\perp}$ is at least two for all points $x, y$ at mutual distance two, then one of the following situations occurs.

i) $(P, L)$ is a nondegenerate polar space of rank $k$ (i.e., a shadow space of type $B_{k, 1}$ ).

ii) $k=3$ and $(P, L)$ is a shadow space of type $A_{n j}$ for some $j, n \in N$ with $1 \leqslant j \leqslant 1 / 2(n+1)<\infty$.

iii) $k=3$ and $(P, L)$ is the quotient of a shadow space of type $A_{2 m+1, m}$ (for some $m$ with $3 \leqslant m<\infty)$ by an involutory automorphism with the property that no point of the shadow space is mapped to a point at distance $\leqslant 4$. The building $B$ associated with the shadow space is defined over an infinite (commutative) field and the automorphism defines a nontype-preserving automorphism of $B$.

iv) $k=4$ and $(P, L)$ is the quotient of a shadow space of type $D_{n, n}$ for some $n$ with $4 \leqslant n<\infty$ by a group of automorphisms such that the identity is the only element mapping a point of the shadow space onto a point at distance $\leqslant 4$. If this group is nontrivial, the associated building is defined over an infinite field.

v) $k=5$ and $(P, L)$ is a shadow space of type $E_{6,1}$.

vi) $k=6$ and $(P, L)$ is a shadow space of type $E_{7,1}$.

Conversely, the spaces in i) ... vi) satisfy the hypotheses if all of their lines have at least three points.

About the proof. For $x \in P$, consider the space $x^{\perp} / x$ whose points are the lines containing $x$ and whose lines are the pencils of lines from $L$ (cf. Section 3). It is a parapolar $(k-1)$-space of diameter $\leqslant 2$.

The polar spaces appearing in $i$ ) are the parapolar spaces which are locally polar spaces (compare Theorem 4.2). Thus, we can reduce to the case where for at least one point $x \in P$ (and hence all points of $P$, cf. Lemma 2 in [23]) the space $x^{\perp} / x$ is a parapolar $(k-1)$-space of diameter $\leqslant 2$ which is not a polar space. The symplecta and complete subspaces provide 'candidate shadow subspaces' for the full shadow space under construction.

For $k=3$, they can be used to define a new parapolar $k$-space with strictly smaller singular rank. By induction on the singular rank this leads to the cases $i i$ ) and $i i i)$. The latter case occurs if the collection of maximal complete subspaces cannot be separated into two parts by the same procedure as described in Section 3 for type $E_{6,4}$. See Cohen [22] for details.

For $k \geqslant 4$, the space $x^{\perp} / x$ can be characterized by induction on $k$. Observe that axiom $(F)_{\{-1,0, k-1\}}$ leads to $(F)_{\{-1, k-2\}}$ for $x^{\perp} / x$, so that the latter must be a shadow space of type $A_{n, 2}$ for some $n$. There are enough 'candidate shadows' available to recognize the space as a quotient of a shadow space of type $D_{n, n}$, whence case $i v$ ). For details, see Brouwer-Cohen [6].

Finally, for $k \geqslant 5$, all 'candidate shadow subspaces' are available and, by the same induction on $k$ as before, the full shadow space corresponding to case $v$ ) or $v i$ ) is easily recognized, see CohenCooperstein [23]

Certain spaces in the above theorem occur locally (i.e., as $x^{\perp} / x$ for some point $x$ ) in other shadow spaces of buildings of spherical type. Thanks to this phenomenon, we can use the above result to derive: 


\subsection{Theorem(Cohen-Cooperstein).}

Let $(P, L)$ be a connected parapolar $k$-space $(k \geqslant 4)$ of finite singular rank all of whose lines have at least three points and in which $(F)_{\{-1,1, k-1\}}$ is satisfied. Then one of the following holds:

i) $(P, L)$ is a nondegenerate polar space of rank $k$.

ii) $k=4$ and $(P, L)$ is a shadow space of type $D_{5,5}$ or $E_{6,4}$.

iii) $k=5$ and $(P, L)$ is a shadow space of type $E_{6,1}$ or $E_{7,7}$.

iv) $k=7$ and $(P, L)$ is a shadow space of type $E_{8,1}$.

Conversely, the spaces in $i), . ., i v)$ satisfy the hypotheses if all of their lines have at least three points.

About the proof. If $x \in P$, the space $x^{\perp} / x$ is a parapolar $(k-1)$-space satisfying $(F)_{\{-1,0, k-2\}}$, so appears in the conclusion of the preceding theorem. Also, since $x^{\perp} \cap S$ cannot be a singleton, the diameter of $x^{\perp} / x$ is at most three. It is easily seen that the isomorphism type of $x^{\perp} / x$ does not depend on the point $x$. Again, $(P, L)$ is a polar space if and only if $x^{\perp} / x$ is a polar space.

Suppose $k=4$. Then $x^{\perp} / x$ is isomorphic to a shadow space of type $A_{4,2}$ or $A_{5,3}$. The first case can be settled as in the previous theorem. There are two difficulties to overcome in finding the full shadow space associated with $(P, L)$ if $x^{\perp} / x$ is of type $A_{5,3}$. First of all, we must find two kinds of maximal complete subspaces, namely the subspaces appearing as shadows of flags of type 2 and 5 , respectively. We have seen before how this can be achieved. Second, the subspaces which are shadows of flags of type 1 and 6 , respectively, are subspaces isomorphic to shadow spaces of type $D_{5,5}$, and hence not yet available from Theorem 5.1. They are defined as the subsets

$$
\bigcup_{y \in X, z \in X-y^{\perp}}\{y, z\}^{\perp}
$$

for $x$ running over all points and $X$ over all subspaces of $x^{\perp}$ containing $x$ with the property that $X / x$ (defined analogously to $x^{\perp} / x$ ) is a subspace of $x^{\perp} / x$ isomorphic to a shadow space of type $A_{4,2}$.

A similar problem arises when $k=5$, for $E_{7,7}$. Here, again, subspaces of type $E_{6,1}$ can be reconstructed by use of local shadows of type $D_{5,5}$. In the last case, $k=7$, no such problem arises.

A fairly immediate consequence of the above theorem is

5.4. Corollary(Cohen-Cooperstein [24]). The shadow spaces of type $D_{n, n}(n \leqslant 7), E_{6,1}$, $E_{6,4}, E_{7,1}, E_{7,7}$, and $E_{8,1}$ are locally recognizable.

\section{Dual polar spaces.}

Polar spaces have been introduced as spaces in which one of the three possible numbers for the rank of $x^{\perp} \cap l$, where $x \in P$ and $l \in L$, never occurred. In this section, the possibility that this intersection is all of $l$ is ruled out whenever $x$ does not belong to $l$. In other words, lines will be maximal complete subspaces. A space $(P, L)$ in which, for each $x \in P$ and $l \in L$ with $d(x, l) \leqslant j$, the set $\pi_{x}(l)$ is a singleton is called a near $2 d$ - gon of depth $j$ if it has diameter $d$.

A 'near $2 d$ - gon of depth $d$ ' is also called a near $2 d$ - gon. Dual polar spaces, i.e., shadow spaces of type $B_{n, n}$, are near $2 n$-gons. A near $2 d$ - gon of depth 2 is very close to being a parapolar 2-space: defining property $i$ ) fails as lines are maximal complete subspaces, and it is the only 
one to fail. In this light, the following beautiful result can be seen as a complement to Theorem 5.1 .

6.1. Theorem(Shult-Yanushka, Brouwer-Wilbrink).

Let $(P, L)$ be a near $2 d$ - gon of depth $j$ (for some $j \geqslant 2$ ) in which lines have at least three points and each pair of points at distance two has at least two common neighbors. Then each pair of points at mutual distance $j$ can be embedded in a unique geodesically closed subspace isomorphic to a near $2 j$ - gon.

About the proof. See Shult-Yanushka [37] for the case where $j=2$ and Brouwer-Wilbrink [7] for the remaining cases.

Applying the above lemma with $j=2$, we obtain subspaces isomorphic to generalized quadrangles. They form the rank 2 counterpart of the symplecta in Theorem 5.1, and are called quads.

\subsection{Theorem(Cameron, Shult, Brouwer-Cohen).}

Suppose $(P, L)$ is a near $2 d$ - gon of depth 3 such that lines have size at least three and each pair of points at mutual distance 2 is contained in a quadrangle. If, for each point $x$ and each quad $S$ with $d(x, S) \leqslant 2$, the set $\pi_{x}(S)$ is a singleton, then, for each $x \in P$, the space of the lines and quads on $x$ is a projective space. If, moreover, this space has finite rank (for some $x \in P$ ), then $(P, L)$ is the quotient of a dual polar space by a group of automorphisms whose nontrivial elements map each point to a point at distance $\geqslant 8$.

In the finite case, the building does not admit nontrivial groups of automorphisms with the specified property, so $(P, L)$ is a shadow space itself (cf. Brouwer-Cohen [6]). The existence of a near 6-gon on 759 points with automorphism group $M_{24}$, due to Shult-Yanushka [37], shows that the condition on $\pi_{x}(S)$ is not superfluous. Obviously, local characterizations are out of the question.

\section{Metasymplectic spaces.}

This section is devoted to shadow spaces of type $F_{4,1}$, also called metasymplectic spaces. The following characterizations are available.

\subsection{Theorem.}

Let $(P, L)$ be a connected parapolar $k$-space $(k \geqslant 3)$ satisfying $(F)_{\mathbf{Z}-\{0\}}$. If every pentagon contains a point $x$ such that $x^{\perp} \cap l$, where $l$ is the line spanned by the two points of the pentagon noncollinear with $x$, is nonempty, then $(P, L)$ is either a polar space of rank $k$ or $k=3$ and $(P, L)$ is a metasymplectic space.

About the proof. The parapolar space condition yields the existence of symplecta, thus leading to sufficiently many 'candidate shadows' for the full shadow space. However, it is not immediate that the geometry whose objects of type 1,2,3,4 are the points, lines, planes, and symplecta, respectively, has diagram $F_{4}$. In particular, it has to be shown that the residue of a point is a dual polar space of rank 3. For details, see Cohen [20], [21]. Due to Axiom $(F)_{z-\{0\}}$, the space $x^{\perp} / x$ has diameter at most 3. The statement about pentagons can be used to apply Theorem 6.2 and derive that $x^{\perp} / x$ is a dual polar space of rank at most 3 . 
In view of the parapolar space axiom iii) and the pentagon axiom, the result is far from a local characterization. There are indications that one might encounter difficulties in trying to prove local recognizability. For instance, the shadow space of the thin building of type $F_{4}$ over 1 is a graph which is locally a cube (points are vertices, lines are edges). By a result due to Buset [17] and (independently) Brouwer there are precisely two graphs which are locally a cube, the 'nonmetasymplectic' one being the complement of the 3 by 5 grid. The local recognizability of metasymplectic spaces with lines of size at least 3 has neither been proved nor disproved. The only result in this direction (known to me) reads as follows.

\subsection{Theorem(Cohen-Cooperstein [24]).}

Let $(P, L)$ be a finite connected space such that for each point $x$ the subset $x^{\perp}$ is a subspace isomorphic to the subspace $x_{1}^{\perp}$ of a shadow space of a thick building of type $F_{4}$ over 1 in which $x_{1}$ is a point. If, for each path $x, y, z, u$ in $P$ with $d(x, z)=d(u, y)=2$ such that the size of $\{x, y, z, u\}^{\perp}$ is at least two, every line of $\{x, y, z\}^{\perp}$ meets $\{x, y, z, u\}^{\perp}$ nontrivially, then $(P, L)$ is a metasymplectic space.

About the proof. The extra condition at the end of the theorem guarantees the possibility of comparing shadows in $x^{\perp} / x$ with shadows in $y^{\perp} / y$ for collinear points $x$ and $y$ in $P$. If $x, z$ are points of $P$ at mutual distance 2, then $\{x, z\}^{\perp}$ induces a subspace $Y$ of the dual polar space $x^{\perp} / x$ which either has no lines or has the property that for each point $y \in Y$ there is a quad $\mathrm{Q}$ with $y^{\perp} \cap Y=y^{\perp} \cap Q$. Now, by use of a theorem of Cameron-Kantor [19], the possibility that such a subspace $Y$ is a generalized hexagon can be excluded (by deriving from the existence a structure for $(P, L)$ very much resembling the complement of the 3 by 5 grid encountered in the thin analogue, and showing that this is absurd by means of a counting argument). This leads to the conclusion that $\{x, z\}^{\perp}$ is either a singleton or a subspace isomorphic to a generalized quadrangle. But then $(P, L)$ is a parapolar 3 -space and we can finish as in the previous theorem.

\section{Some remaining points of interest and lines of progress.}

\subsection{Simplify and unify the present set of axioms.}

In his thesis, Hanssens [29] has combined several of the aforementioned results and characterized almost all shadow spaces of buildings of spherical type. Here we state only one of his theorems. It uses an axiom of Buekenhout [9] concerning intersections of symplecta.

\subsubsection{Theorem(Hanssens).}

Let $(P, L)$ be a connected parapolar $k$-space $(k \geqslant 3)$ of finite singular rank all of whose lines have at least three points and in which $x^{\perp} / x$ is connected for each $x \in P$. If the ranks of every pair of maximal complete subspaces differ by at most 1 and no two symplecta meet in a complete subspace of rank $k-2$, then $(P, L)$ is a shadow space of type $A_{4,2}, B_{n, 1}, D_{5,5}, E_{6,1}, E_{7,1}, E_{8,1}$, or $F_{4,1}$.

Thus, a shadow space for each building of spherical type and rank at least three occurs in the conclusion, except for the types $A_{n}(n \geqslant 5)$. The latter types would appear if the requirement about the difference between ranks of maximal complete subspaces were dropped. By the way, if we discard this condition for $k<5$, the theorem remains true provided all spaces occurring in the conclusion of Theorem 5.2 are added to the conclusion of the present theorem. As for $k=2$, Theorem 6.2 fits into the present context if the condition on $\pi_{x}(S)$ is replaced by the equivalent 
condition that the intersection of no two quads is a singleton.

We have seen that Part $i v$ ) of Theorem 2.1 motivates the choice of parapolar spaces as a starting point for characterizations of shadow spaces. Certain special cases of Part iii) of this theorem have been used above. A parapolar space in which iii) holds, for X running over complete subspaces and/or symplecta, might be an appropriate setting for uniform characterization theorems of shadow spaces of spherical type.

\subsection{Weakening the hypotheses.}

The above theorems could be further examined with regard to possible relaxations of conditions on line length, nondegeneracy, finiteness of singular rank, etc. We mention a few results in this direction.

\subsubsection{Polar spaces.}

The polar spaces with arbitrary line size (the weak buildings of type $B_{n, 1}$ ) are completely classified by Buekenhout-Sprague [16], and degenerate polar spaces are dealt with in JohnsonShult [30], but little structure theory is available if the rank is not assumed to be finite.

As we have seen in Theorem 2.1, nondegenerate polar spaces are determined by their collinearity graph. In this context, it may be worth mentioning that a large portion of the structure theory for these collinearity graphs in Buekenhout-Shult [15] carries over to a more general class of graphs $(P, \perp)$ satisfying the single axiom:

For every maximal complete subgraph $C$ and every pair $x, y$ of vertices, the inclusion $x^{\perp} \cap C \subseteq y^{\perp} \cap C$ implies $x^{\perp} \cap C=y^{\perp} \cap C$ or $y \in P^{\perp}$.

This observation, due to Hall \& Wilbrink, generalizes earlier work by Zara [42], who already knew that the graph on 275 vertices associated with the McLaughlin group, whose maximal complete subgraphs have size 5 , is a sporadic example.

\subsubsection{Parapolar spaces.}

In Theorem 5.2, there are (at least) four additional examples satisfying all conditions (with $k=3$ ) except for the line length:

number of
points
36
176
378
22880

intersection
array
$(15,8 ; 1,6)$
$(40,27 ; 1,8)$
$(45,32,12,1 ; 1,6,32,45)$
$(280,243,144,10 ; 1,8,90,280)$
associated group
$\mathrm{Sz}$

$$
\begin{gathered}
\text { vertex } \\
\text { stabilizer } \\
S_{6} \\
P S U_{4}(2) \cdot 3 \\
\Omega_{5}(3) \\
3 \cdot P S U_{4}(3) \cdot 2
\end{gathered}
$$

reference

In fact, any graph which is locally a generalized quadrangle of order $(s, t)$ and in which $\{x, y\}^{\perp \perp}$, for two points $x, y$ at mutual distance two induces a complete bipartite graph on $2(t+1)$ points, is a parapolar 3 -space satisfying $(F)_{\{-1,0,2\}}$ all of whose lines have size two. Here, the line length (two) of the space is not to be confused with the line length $(s+1)$ of the generalized quadrangle $x^{\perp} / x$ for a point $x$. (In fact, $s+2$ is the size of a maximal complete subspace.) It is as yet an open problem whether the above four are the only examples apart from those in conclusions $i$ ), $i i$ ) and $i i i$ ) of the theorem. (As for $i i i$ ), the shadow space of the thin building of type $A_{2 m+1}$ over $m$ admits the same kind of quotients as the infinite buildings.) This question is part of the general problem of classifying all graphs which are locally generalized quadrangles formulated in Buekenhout-Hubaut [13]. 
There is also an example of a parapolar 4-space on 117 points satisfying all conditions of Theorem 5.2 except the one on the line size, which is locally the above graph on 36 vertices, see Hall-Shult $[28]$.

\subsubsection{Dual polar spaces.}

In view of Brouwer-Wilbrink [7] and Brouwer-Cohen [6], every finite near 8 - gon with the property that each pair of points at mutual distance two is contained in a thick quad (i.e., a shadow space of a thick building of type $B_{2}$ over 1 ) is a dual polar space if it satisfies certain regularity conditions. In other words, under these circumstances one can dispense with the condition on $\pi_{x}(S)$ in Theorem 6.2. Apart from $M_{24}$, there is another sporadic finite simple group, namely HJ, associated with a near $2 d$ - gon; $d=4$, there are 315 points and no quads.

\subsubsection{Metasymplectic spaces.}

In Tits [40] (cf. p.216), the problem of determining all metasymplectic spaces associated with weak buildings is mentioned. As of today, this relatively easy (?) problem has not been worked out. In Theorem 7.1, finiteness of singular rank is not assumed. It is obscure (to me) what the implications are of relaxing the condition that $l^{\perp \perp}$ is not a complete subspace.

In the same vein as the problem of Buekenhout-Hubaut, there is the problem of classifying all graphs which are locally dual polar spaces. Graphs related to the sporadic groups $F_{1}$ (the Monster) and $F_{24}$ (Fischer's group) exist which are locally dual polar spaces of rank 3, cf. Buekenhout-Fischer [12] and Ronan-Stroth [35]. (This can be read off from the relevant diagrams.)

\subsection{Embedding problems.}

The classification of buildings of type $B_{n}$ and $D_{n}(n \geqslant 3)$ of Tits and Veldkamp (see [39]) heavily employs embeddings of the associated polar space $(P, L)$ in a projective space $\left(P_{0}, L_{0}\right)$ (i.e., a pair of injective mappings $P \rightarrow P_{0}$ and $L \rightarrow L_{0}$ such that for every line in the image all of its points belong to the image of $P$ ). In fact, Tits and Veldkamp considered a somewhat more restrictive notion of embedding. Buekenhout-Lefêvre [14] and Dienst [27] (see also [31]) showed that the only generalized quadrangles embeddable in projective space are the 'classical' embeddable ones. Putting all this together, we know for each polar space of rank at least two into which projective spaces (of rank at least three) it can be embedded. It turns out that, in a sense, there is always a universal embedding. Now, the analogous problem, with the 'projective spaces' and 'polar spaces' replaced by other shadow spaces, has been given little attention so far. The reader is referred to A. Wells [41] for the most recent result in this context. Since the shadow spaces of spherical type are known and heavily related to groups, it makes sense to study the problem of embeddings by means of representation theory of the groups involved. In Ronan-Smith [34], such an approach can be found.

\subsection{Local recognition.}

The conditions in Theorem 8.1.1 imposed on the maximal complete subspaces and symplecta are local in nature (since the intersections of symplecta are already determined by local data). In view of this and foregoing theorems, the question arises which parapolar spaces are locally connected (or even locally the shadow of a building) but not covered by a shadow space (i.e., a quotient of a shadow space by a judiciously chosen group of automorphisms mapping each point to a point at distance at least four). More generally, one might ask for each shadow space $(P, L)$ : What is the minimal number $k$ such that each space $\left(P_{1}, L_{1}\right)$ is covered by $(P, L)$ if it has the 
property that, for each $y_{1} \in P_{1}$, the set $\left\{x_{1} \in P_{1} \mid d\left(x_{1}, y_{1}\right) \leqslant k\right\}$ is a subspace isomorphic to $\{x \in P \mid d(x, y) \leqslant k\}$ for some $y \in P$ ? Some partial answers, in particular for $k=1,2$, have been given above. It seems that if the diameter of $x^{\perp} / x$ is two or three, $k$ will be small as well. Other local recognitions appear in Hall-Shult [28].

\subsection{Analogues of affine space.}

The classical affine space can be obtained by removing a hyperplane from a projective space. There is a natural extension to other shadow spaces: A proper subspace of a space is called a hyperplane if each line meets it nontrivially. For projective spaces, this definition of hyperplane coincides with the usual one. A hyperplane without lines in a generalized quadrangle is usually called an ovoid. Taking for $P$ the set of all vectors of a given vector space $V$ and for $L$ the set of all affine lines $l$ in $V$ such that $Q(x-y)=0$ for all $x, y \in l$, where $Q$ is a quadratic form on $V$ (or one of the obvious analogues), we obtain an 'affine polar space' $(P, L)$ in the intuitive sense. It is also an example in the formal sense that it can be obtained as the space whose point set is the complement $A$ of a hyperplane in a polar space, and whose lines are the sets $l \cap A$, for $l$ running over all lines of the polar space for which $l \cap A$ has size at least two.

Nice axiomatizations of affine space (almost in terms of diagram geometry, cf. Buekenhout [8]) have been given. What extensions to other shadow spaces do exist? For polar spaces, this problem is under study and a solution seems within reach.

\subsection{Application to group theory.}

The only application of the above theory to the classification of finite simple groups (known to me) can be found in Aschbacher [1], where it is also mentioned that it is possible to do without. Nevertheless, at p.463, one can find reference to another possible application. In fact, it would be rather natural to try and replace part of the classification of Chevalley groups as groups generated by root subgroups (see, e.g., Timmesfeld [38]) by geometric arguments such as those occurring in the proof of Theorem 5.3.

\subsection{Shadow spaces of buildings of affine type.}

In view of Theorem 2.1, it is tempting to extend parapolar space theory to the case where $\{x, y\}^{\perp}$ for $x, y \in P$ is either a projective space or a nondegenerate polar space. As yet, little has been done in this direction. I finish with the first (?) characterization of shadow spaces of buildings of affine type. The proof, by the way, is easily derived by the methods described above. Denote by $r$ the node of extension in the Coxeter diagram $E_{9}=\tilde{E}_{8}$.

\subsubsection{Theorem.}

$A$ shadow space of type $E_{9, r}$ is 'almost' locally recognizable up to quotients in the following sense: A parapolar 8-space in which for each point $x$ the set $x^{\perp}$ is a subspace isomorphic to the subspace $y^{\perp}$ of a shadow space of type $E_{9, r}$ (containing the point $y$ ) is isomorphic to the quotient of a shadow space of type $E_{9, r}$ by a group of automorphisms whose nontrivial elements map each point to a point at distance at least five. 


\section{References}

[1] Aschbacher, M., A characterization of Chevalley groups over fields of odd characteristic, Annals of Math. 106 (1977) 353-468.

[2] Biggs, N.L. Algebraic Graph Theory, Cambridge University Press, Cambridge, 1974.

[3] Blokhuis A. \& A.E. Brouwer, private communication.

[4] Bourbaki, N., Groupes et algèbres de Lie, Chap. IV, V, VI, Hermann, Paris, 1968.

[5] Brouwer, A.E. \& A.M. Cohen, Some remarks on Tits geometries, Indagationes Mathematicae 45 (1983)

[6] Brouwer, A.E. \& A.M. Cohen, Local recognition of some Tits geometries of classical type, to appear in Geom. Dedicata.

[7] A.E. Brouwer \& H.A. Wilbrink, The structure of near polygons with quads, Geom. Dedicata 14(1983) 145-176. Amsterdam, 1981.

[8] Buekenhout, F., Une caractérisation des espaces affines basée sur la notion de droite, Math. Zeitschr. 111 (1969) 367-371.

[9] Buekenhout, F., An approach to building geometries based on points, lines and convexity, European J. Combinatorics 3 (1982) 103-118.

[10] Buekenhout, F., A characterization of polar spaces, Simon Stevin 53 (1973) 3-7.

[11] Buekenhout, F., Cooperstein's Theory, Simon Stevin 57 (1983) 125-140.

[12] Buekenhout, F. \& B. Fischer, A locally dual polar space for the Monster, preprint.

[13] Buekenhout, F. \& X. Hubaut, Locally polar spaces and related rank 3 groups, J. Algebra 45 (1977) 393-434.

[14] Buekenhout, F. \& C. Lefèvre, Generalized Quadrangles in projective spaces, Arch. Math. 25 (1974) 540-552.

[15] Buekenhout, F. \& E.E. Shult, On the foundation of polar geometry, Geom. Dedicata 12 (1982) 75-85.

[16] Buekenhout, F. \& A. Sprague, Polar spaces having some line of cardinality two, J. Combinatorial Theory (A) 33 (1982) 223-238.

[17] Buset, D., Graphs which are locally a cube, Discrete Math. 46 (1983) 221-226.

[18] Cameron, P.J., Dual polar spaces, Geom. Dedicata 12 (1982) 75-85.

[19] Cameron, P.J. \& W.M. Kantor, 2-Transitive and antiflag transitive collineation groups of finite projective spaces, J. Algebra 60 (1979) 384-422.

[20] Cohen, A.M., An axiom system for metasymplectic spaces, Geom. Dedicata 12 (1982) 417433.

[21] Cohen, A.M., On the points and lines of metasymplectic spaces, Annals of Discrete Math. 18 (1983) 193-196.

[22] Cohen, A.M., On a theorem of Cooperstein, European J. Combinatorics 4 (1983) 107-126.

[23] Cohen, A.M. \& B.N. Cooperstein, A characterization of some geometries of exceptional Lie type, Geom. Dedicata 15 (1983) 73-105.

[24] Cohen, A.M. \& B.N. Cooperstein, On the local recognition of finite metasymplectic spaces, preprint. 
[25] Cooperstein, B.N., Some geometries associated with parabolic representations of groups of Lie type, Canadian J. Math. 28 (1976) 1021-1031.

[26] Cooperstein, B.N., A characterization of some Lie incidence structures, Geom. Dedicata 6 (1977) 205-258.

[27] Dienst, K.J., Verallgemeinerte Vierecke in projektiven Räumen, Arch. Math. 35 (1980) 177 186.

[28] Hall, J.I. \& E.E. Shult, Locally cotriangular graphs, to appear.

[29] Hanssens, G.J.J.J., Punt-rechte meetkunden van sferische gebouwen, Thesis, R.U. Gent, 1984.

[30] Johnson, P. \& E.E. Shult, Local characterizations of polar spaces, to appear in Geom. Dedicata.

[31] Lefèvre-Percsy, C., Polar spaces embedded in projective space, pp.216-220 in: Finite Geometries and Designs, (P.J. Cameron, J.W.P. Hirschfeld, D.R. Hughes, eds.) LMS Lecture Note Series 49, Cambridge University Press, Cambridge, 1981.

[32] Ronan, M.A., Extending locally truncated buildings and chamber systems, to appear.

[33] Ronan, M.A., Coverings of certain finite geometries, pp.316-331 in: Finite Geometries and Designs, (P.J. Cameron, J.W.P. Hirschfeld, D.R. Hughes, eds.) LMS Lecture Note Series 49, Cambridge University Press, Cambridge, 1981.

[34] Ronan, M.A. \& S.D. Smith, Sheaf homology on buildings and modular representations of Chevalley groups, I,II,III, preprints.

[35] Ronan, M.A. \& G. Stroth, Minimal parabolic geometries for the sporadic groups, European J. Combinatorics 5 (1984) 59-91.

[36] Shult, E.E., Lie incidence geometries, pp.157-184 in:Surveys in Combinatorics, E. Keith Lloyd (ed.), London Math. Soc. Lecture Note Series 82, Cambridge University Press, Cambridge, 1983.

[37] Shult, E.E. \& A. Yanushka, Near n-gons and line systems, Geom. Dedicata 9 (1980) 1-72.

[38] Timmesfeld, F., Groups generated by root involutions I,II, J. Algebra 33 (1975) 75-135, 35 (1975) 367-441.

[39] Tits, J., Buildings of Spherical Type and Finite BN-pairs, Springer Lecture Notes in Math. 386, Springer, Berlin, 1974.

[40] Tits, J., A local approach to buildings, pp. 519-547 in : The Geometric Vein, Ch. Davis et al. (eds.), Springer, Berlin, 1981.

[41] Wells, A.L., Universal projective embeddings of the Grassmannian, half spinor, and dual orthogonal geometries, Quart. J. Math. Oxford (2) 34 (1983) 375-386.

[42] Zara, F., Graphes lie's aux espaces polaires, preprint. 